\title{
Efficacy and Safety of Prophylactic Intrathecal Normal Saline for Prevention of Post Dural Puncture Headache among Women Undergoing Cesarean Section under Spinal Anesthesia: A Randomized Controlled Trial
}

Semagn Abate ( $\nabla$ semmek17@gmail.com )

Dilla University https://orcid.org/0000-0001-5661-8537

\section{Siraj Ahmed}

Dilla College: Dilla University

\section{Getachew Mergia Anbese}

Dilla College: Dilla University

\section{Research}

Keywords: PDPH, Normal Saline, Spinal Anesthesia, Intrathecal

Posted Date: June 16th, 2021

DOl: https://doi.org/10.21203/rs.3.rs-584207/v1

License: (1) (i) This work is licensed under a Creative Commons Attribution 4.0 International License. Read Full License 


\section{Abstract}

Background: Post-Dural Puncture Headache is the commonest complication of spinal anesthesia. Body of evidence revealed that Conservative management failed to show significant benefit and epidural needle and catheter techniques didn't provide conclusive evidence. On the other hand, intrathecal injection of normal saline is a simple technique and cost-effective in a resource-limited setup but it is not well examined on its effectiveness and safety profiles.

Methods and materials: After Obtaining Ethical clearance from IRB, 152 mothers scheduled for cesarean section under spinal anesthesia were allocated randomly into two groups. Data analysis was done with SPSS version 22. Descriptive statistics were run to see the overall distribution of the study subjects. Unpaired student's T-test for continuous symmetric data and Mann-Whitney $U$ test for non-normally distributed data were used. Categorical data were analyzed with Chi-square and fisher's exact test where appropriate. A generalized estimating equation model was used to investigate the interaction of repeated measurements of NRS pain scores of PDPH.

Result: The overall incidence of PDPH was $29.6 \%$ while the proportion of patients who experienced PDPH was the highest among patients with control as compared to intervention( $36.8 \%$ vs $22 \%$ ) respectively. The GEE model revealed that the NRS pain score was $0.7,0.4$, and 0.2 unit higher at 12,24 , and 48 respectively in control as compared to the intervention

Conclusion: prophylactic intrathecal normal saline could be an option in a resource-limited setup where the appropriate spinal needle is not accessible and management of moderate and severe PDPH is not feasible

Registration: The protocol was registered prospectively in Clinical Trials.gov (NCT04393766).

\section{Introduction}

The global rate of cesarean section is increasing despite the World Health organization's recommendation rate of cesarean section( 1,2$)$. According to a WHO report, more than 18 million cesarean sections are performed every year(1).

Approximately, $80-90 \%$ of cesarean sections are performed with spinal anesthesia which is relatively safe as compared to general anesthesia which is accompanied by a difficult airway and risk of aspiration(3-6). However, post-dural puncture Headache is one of the commonest complications of the neuraxial block associated with penetration of dura matter and continuous flow of cerebrospinal fluid (714).

The International Headache Society (IHS) defines PDPH as "Headache occurring within 5 days of a lumbar puncture caused by cerebrospinal fluid (CSF) leakage through the dural puncture. It worsens 15 minutes of standing or sitting and improves after 15 minutes of lying down. It is usually accompanied by 
neck stiffness and/or subjective hearing symptoms, nausea and vomiting, photophobia. It recovers spontaneously within one week, or after sealing of the leak with an autologous epidural lumbar patch within 48 hrs. There is no fever, leukocytosis, and neurologic deficit(15)."

Ninety percent of postural puncture headaches will occur within three days of dural puncture whereas sixty-six percent of the headache may happen within $48 \mathrm{hrs}$ of the procedure and rarely, the headache will develop between five to fourteen days after the procedure. However, some reports showed cases of postdural puncture within 20 minutes of dural puncture and 19 months after dural puncture which were successfully managed with Epidural Blood Patch(9).

The exact mechanism of PDPH is still unknown but symptoms are generally attributed to excessive loss of CSF from the dural puncture site which results in reduced CSF pressure(12).

The lowered CSF pressure reduces the cushioning effect provided by the CSF to the brain and results in traction on intracranial pain-sensitive structures such as meningeal vessels, upper cervical and cranial nerves. The release of adenosine consequent to the sudden drop in CSF volume is also thought to cause vasodilatation of intracranial vessels and post-dural puncture headache(4).

Different factors of post-dural puncture have been mentioned in the literature among which needle size and volume of cerebrospinal fluid (CSF) lost were the independent predictors of severity of PDPH after spinal anesthesia, diagnostic lumbar puncture, or accidental dural puncture during epidural procedures(4, $7-12,14,16-24)$. The incidence of post-dural puncture headache is more likely in adults whose ages are between 30-50 years as compared to older age greater than 50 and children younger than 13 years old.

The lower weight is found to be strongly associated with the higher incidence of PDPH and cumulating evidence showed an inverse relationship between BMI and PDPH suggesting that heavier patients, in general, have higher intra-abdominal pressure, which in turn raises intra-epidural pressure and prevents cerebrospinal fluid from leaking when $\operatorname{ADP}$ occurs $(4,25)$.

Different studies showed that smokers had a considerably reduced rate of PDPH in comparison with nonsmokers suggesting an inhibitory effect of tobacco smoking on PDPH that may be associated with the stimulation role of nicotine in dopamine neurotransmission $(4,15,26)$.

Though pregnancy is a risk factor for PDPH due to the young age, female, sometimes sitting position, pregnancy-associated depression and anxiety, and the special popularity of regional anesthesia in this population, a meta-analysis showed that pregnancy itself does not increase the risk of PDPH. However, PDPH is associated with fatigue, sleep deprivation, and night work that lead to a higher incidence of ADP in clinical personnel when performing epidural analgesia(4).

Evidence showed that the prevalence of post-dural puncture headache in Ethiopia after spinal anesthesia was very high ranges from $38.7-42.6 \%(18,23)$. While other studies conducted in Sub-Saharan Africa showed that the prevalence of PDPH varied from 15 to $27.5 \%$ which was strongly correlated with needle type and size $(17,27-31)$. 
The incidence of PDPH with a randomized clinical trial conducted in Egypt on ninety obstetric patients in three groups receiving single-shot spinal anesthesia, continuous spinal anesthesia with epidural Cather and single-shot spinal with prophylactic $5 \mathrm{ml}$ normal saline and $10 \mathrm{ml}$ normal saline before removal of the catheter after twenty-four hours was $10 \%, 16.6 \%$, and $10 \%$ respectively(32).

A systemic review conducted in the United Kingdom revealed that the incidence of post-dural puncture headache after spinal anesthesia varied from $1.5 \%-11 \%$. Whereas, $50 \%$ of patients developed post-dural puncture headache after accidental dural penetration with 16-18 gauges epidural needle(21).

The Cochrane database for systemic reviews identified incidence of post-dural puncture headache as $10 \%$ after spinal anesthesia, $36 \%$ after diagnostic lumbar puncture, and $81 \%$ after accidental dural puncture(9).

PDPH is getting worse with movement which leads to different undesirable consequences including delayed mother-to-child bonding, delayed breastfeeding, prolonged hospitalization, deep venous thrombosis, and thromboembolism incidents, delayed oral intake, and increase health care system $\operatorname{cost}(33)$.

An epidural blood patch is the known gold standard management PDPH for years when conservative management failed to be effective despite inconclusive evidence as depicted with recent systemic reviews and meta-analysis $(34,35)$.

Different systemic reviews and meta-analyses and randomized controlled trials revealed that conservative management for PDPH including bed rest, oral fluid intake, simple oral analgesics, and caffeine failed to show significant benefit and was associated with undesirable side effects $(11,21,34$, 36-38).

A systemic review and meta-analysis by Apfel et al identified inconclusive evidence from one study with subgroup analysis. However, the quality of the included study for subgroup analysis had a small sample size, no randomization, no allocation concealment, no blinding, and proper controlling of confounders were not considered as a result both the original RCT and the systemic review recommends further RCT with high power and proper randomization(34).

A randomized clinical trial conducted to investigate the preventive benefits of intrathecal normal saline revealed a statistically significant difference in the severity and prevalence of PDPH between the groups. However, this RCT failed to assess adverse effects associated with administration of normal saline including inadequate sensory and motor block, and high spinal block and they recommend further RCT with large sample size and possible adverse effects(39).

Despite recent advancements in neuraxial techniques and different clinical and epidemiological researches on the treatment and prevention of post-dural puncture headache, the incidence of PDPH is very high and further randomized trials are in demand on its prevention approaches. Therefore, this study 
aimed to investigate the efficacy and safety of prophylactic intrathecal normal saline for the prevention of PDPH after spinal anesthesia during cesarean delivery

\section{Materials And Methods}

\subsection{Protocol and registration}

This parallel double-blinded prospective randomized controlled study was conducted at Dilla University Referral Hospital from January 2020 to April 2021. Ethical clearance was obtained from Dilla University college of health sciences Institutional Review Board and it was given a Unique Identifier number(UIN:0014/19 - 11). The protocol was registered prospectively Clinical Trials.gov (NCT04393766). The objective of the study was explained and written informed consent was obtained from each participant by the data collector. Confidentiality was maintained at all levels of the study by avoiding identifiers and using codes to identify participants. The findings of the study were reported in compliance with Consolidated Standards of Reporting Trials (CONSORT) statement guidelines for randomized controlled trials(40).

\subsection{Participants}

Parturient who were term pregnant, ASA I and II, aged 18 to 35 years old, scheduled for cesarean section under spinal anesthesia and agreed to sign the informed consent form after comprehending the objectives of the study were included. Parturient with a history of migraine, history of PDPH, and BMI $>25$ $\mathrm{kg} / \mathrm{m}^{2}$ were excluded.

\subsection{Sample size determination}

The required sample size was calculated using the incidence of PDPH from a previous study ( $16 \% \mathrm{VS}$ $2 \%$ ) with $G$ power software version 3.1.9.2 with the assumption of $80 \% \operatorname{power}(\beta=0.2)(47)$ that can bring a significant difference between the groups at $95 \%$ confidence interval and $5 \%$ significance level. This gave a sample size of 152 along with $10 \%$ for noncompliance.

\subsection{Randomization and intervention}

On the morning of surgery, every patient was re-evaluated for identification, baseline vital sign, laboratory investigation, and informed consent. Eligible patients were randomized into either treatment or control group randomly with a random sequence generated list of numbers and code which was computed with R package (Random Allocation Rule function) version 3.6.0 by the research assistant as per the schedule number given by the preoperative assessor. The list of randomly generated numbers along with the code was sealed with an opaque envelope which was later opened by the anesthetist not involved in the patient care. The data collector and the patient were blinded to the type of intervention. As per routine spinal anesthesia protocol, Group1 received $5 \mathrm{ml}$ intrathecal Normal saline followed by $12.5 \mathrm{mg}$ of hyperbaric $0.5 \%$ Bupivacaine and Grup2 received only $12.5 \mathrm{mg}$ hyperbaric $0.5 \%$ bupivacaine (Fig. 1 ).

\subsection{Data collection tools and procedures}


Data collection was carried out with pretested questionnaire which comprises Socio-demographic characteristics, preoperative patient clinical parameters, intraoperative and postoperative patient clinical variables. Data were collected with two senior Anesthetists who are not involving inpatient care. Preoperatively, the patient's age and body mass index along with the patient's baseline heart rate respiratory rate, and blood pressure were taken. Intraoperatively, sensory onset, peak sensory block were assessed every two minutes with pinprick until the desired level is achieved (T6-T4), and Motor block was assessed with a revised Bromage scale(41).

\subsection{Outcomes and follow up}

The primary outcomes of interest were incidence and severity of PDPH which were measured postoperatively at $12 \mathrm{hrs}, 24 \mathrm{hrs}, 48 \mathrm{hrs}, 72 \mathrm{hrs}$, and 5 th day. Most of the patients were discharged from the hospital after $48 \mathrm{hrs}$ but the remaining data at $72 \mathrm{hrs}$ and 5 th day were received with telephone contact. The secondary outcomes include sensory onset, peak sensory level, and degree of motor block, intraoperative systolic blood pressure, diastolic blood pressure, mean arterial blood pressure, heart rate, and arterial oxygen saturation every five minutes for thirty minutes. Besides, the neonatal Apgar scores, and complications related to spinal anesthesia and PDPH. Postoperatively, the incidence of post-dural puncture headache was assessed and if any, the severity was assessed with eleven points Numeric Rating Scale (NRS) at $12 \mathrm{hrs}, 24 \mathrm{hrs}, 72 \mathrm{hrs}$, and 5 th day. Nausea and vomiting were assessed while assessing PDPH with the Numeric Rating scale.

\subsection{Data processing and Analysis}

Data were entered, cleaned by Epi-info version 7, and imported to Statistical Package for Social Sciences version 22 for analysis. Descriptive statistics were run to see the overall distribution of the data. Unpaired student's T-test for continuous symmetric data and Mann-Whitney $\mathrm{U}$ test for non-normally distributed data were used. Categorical data were analyzed with Chi-square and fisher's exact test where appropriate. A generalized estimating equation model was used to investigate the interaction of repeated measurements of NRS pain scores of PDPH. The finding was reported based on the guidelines of the Consort statement. P-Value of less than 0.05 was considered as significantly associated with the outcome variable.

\section{Result}

\subsection{Sociodemographic characteristics}

As per our operational definition, the majority of participants were ASA I and most of whom had at least one cesarean section. The age of the participants varied from 18 to 38 years with a mean ( \pm SD) of 26.3 \pm 4.21 years while the weight was in the range of 50 to 102 kilogram with a mean $( \pm$ SD) of $72.04 \pm 9.9$ kilograms (Table 1). 
Table 1

Sociodemographic characteristics of participants who underwent cesarean section under spinal anesthesia in DURH, SNNPR, Ethiopia, 2021

\begin{tabular}{|llll|}
\hline Variables & Normal saline & Bupivacaine alone & P-value \\
\hline Age $($ years $)$ & $26.09 \pm 3.53$ & $26.52 \pm 4.81$ & $0.03^{*}$ \\
\hline Weight $(\mathrm{kg})$ & $73.92 \pm 11.28$ & $70.80 \pm 8.41$ & $>0.05$ \\
\hline Height $(\mathrm{cm})$ & $163 \pm 5.84$ & $162.22 \pm 5.88$ & $>0.05$ \\
\hline BMI $\left(\mathrm{kg} / \mathrm{m}^{2}\right)$ & $27.66 \pm 4.46$ & $26.98 \pm 3.53$ & $>0.05$ \\
\hline ASA status & 11 & 65 & $>0.05$ \\
ASA I & 65 & 63 & \\
ASA II & & & \\
\hline Parity & 18 & 58 & $>0.05$ \\
Nulliparous & 29 & 47 & \\
Multiparous & 40 & 32 & \\
\hline Previous cesarean section & 36 & 36 & \\
Yes & & & \\
\hline No & 40.05 & \\
\hline $\begin{array}{l}\text { Note: ** very significant; *significant; ASA: American Society of Anesthesiology; BMI: Body Mass } \\
\text { Index }\end{array}$ & & & \\
\hline
\end{tabular}

\subsection{Preoperative patient data}

This study showed that the majority of indications for cesarean section was previous cesarean section scar $52(34.2 \%)$ followed by cephalopelvic disproportion $46(30.3 \%)$, Oligohydraminos $23(15.1 \%)$, and fetal distress $15(9.7 \%)$ respectively. The baseline hemodynamic parameter between the groups was comparable (Table 2). 
Table 2

preoperative diagnosis and hemodynamic parameters of participants who underwent cesarean section under spinal anesthesia in DURH, SNNPR, Ethiopia, 2021

\begin{tabular}{|llll|}
\hline Variables & Normal saline & Bupivacaine alone & P-value \\
\hline Indication for cesarean section & & & \\
Previous cesarean section scar & $27(51.9)$ & $25(48.1)$ & $>0.05$ \\
CPD & $26(56.5)$ & $20(43.5)$ & $>0.05$ \\
Oligohydramnios & $12(52.2)$ & $11(47.8)$ & $>0.05$ \\
PROM & $2(33.3)$ & $4(99.7)$ & $>0.05$ \\
Malprsentation & $3(37.5)$ & $5(62.5)$ & $>0.05$ \\
Fetal distress & $6(35.3)$ & $11(64.7)$ & $>0.05$ \\
\hline Hematocrit (\%) & $39.59 \pm 2.67$ & $39.32 \pm 2.79$ & $>0.05$ \\
\hline Respiratory rate & $16.04 \pm 2.29$ & $17.01 \pm 4.80$ & $>0.05$ \\
\hline Heart rate & $95.78 \pm 11.91$ & $97.07 \pm 14.80$ & $>0.05$ \\
\hline SBP & $125.53 \pm 10.28$ & $124.32 \pm 8.57$ & $>0.05$ \\
\hline DBP & $77.78 \pm 5.38$ & $78.50 \pm 4.79$ & $>0.05$ \\
\hline MAP & $109.61 \pm 7.87$ & $109.04 \pm 6.48$ & $>0.05$ \\
\hline SPO2 & $97.34 \pm 1.11$ & $97.34 \pm 0.95$ & $>0.05$ \\
\hline $\begin{array}{l}\text { Note: CPD: Cephalopelvic disproportion; PROM: Premature Rupture of Membrane; SBP: Systolic Blood } \\
\text { Pressure; DBP: Diastolic Blood Pressure; MAP: Mean Arterial Blood Pressure; SPO2: percutaneous } \\
\text { Oxygen saturation }\end{array}$ & & \\
\hline S & & & \\
\hline S IntraO & & & \\
\hline
\end{tabular}

\subsection{Intraoperative variables}

The study revealed that the time of spinal drug injection to skin incision, uterine incision, baby out time, sensory onset; surgery duration, anesthesia duration, Apgar scores, and hemodynamic parameters didn't show a significant mean difference between the groups. Besides, the sensory onset and degree of the motor block didn't show a significant difference between the groups (supplemental Table 1).

\subsection{Incidence of post-dural puncture headache}

The overall incidence of post-dural puncture was $29.6 \%$ while the proportion of patients who experienced post-dural puncture headache during five-day follow-up was the highest among patients with bupivacaine alone as compared to patients with bupivacaine and Normal saline, $36.8 \%$ vs $22 \%$ respectively. Despite a clinically significant difference, there was no statistical difference between the group on the incidence of post-dural puncture headache, $p>0.05$ (Fig. 2) 
The incidence of PDPH was the highest at 12 and 24 hrs between the group during the five-day follow-up period which had a significant difference at a p-value of $<0.05$. However, the incidence of PDPH was almost comparable between the group at $48 \mathrm{hrs}$ and thereafter (Table 3 ).

Table 3

The proportion of patients who developed PDPH during follow-up among participants who underwent cesarean section under spinal anesthesia in Dilla University referral hospital, SNNPR, Ethiopia, 2021

\begin{tabular}{|llllll|}
\hline \multirow{2}{*}{ Time } & \multicolumn{2}{l}{ Normal Saline } & \multicolumn{2}{l}{ Bupivacaine alone } & \multirow{2}{*}{ P-value } \\
\cline { 2 - 5 } & PDPH & No PDPH & PDPH & No PDPH & \\
\hline At 12hrs & 9 & 67 & 19 & 57 & $0.036^{*}$ \\
\hline At 24hrs & 10 & 66 & 20 & 56 & $0.042^{\star}$ \\
\hline At 48hrs & 6 & 70 & 8 & 68 & $>0.05$ \\
\hline At 72hrs & 6 & 70 & 2 & 74 & $>0.05$ \\
\hline At 84hrs & 3 & 73 & 4 & 72 & $>0.05$ \\
\hline NOTE: ** Very significant; *significant; PDPH: Post Dural Puncture Headache \\
\hline
\end{tabular}

The severity of PDPH was measured with a Numeric rating scale at each follow-up period. The study revealed that the mean severity score was the highest for the first 48hrs and decreasing thereafter in both intervention and control group (Fig. 3)

\subsubsection{Repeated measure interaction}

The generalized Estimating Equation (GEE) model was employed to investigate the interaction of NRS pain score rating repeated measurements during the five-day follow-up. The GEE model was fitted after restructuring the NRS pain score for each measurement interval. The GEE model revealed that the NRS pain score was $0.7,0.4$, and 0.2 unit higher at 12,24 , and 48 respectively in the Bupivacaine group as compared to the Normal Saline group (Table 5). 
Table 4

Repeated measure interaction estimation with generalized estimation equation for NRS pain score at different periods during follow up

\begin{tabular}{|c|c|c|c|c|c|c|c|}
\hline \multirow[t]{2}{*}{ Parameter } & \multirow[t]{2}{*}{$\beta$} & \multirow[t]{2}{*}{ Std. Error } & \multicolumn{2}{|c|}{ 95\% Wald Confidence Interval } & \multicolumn{3}{|l|}{ Hypothesis Test } \\
\hline & & & Lower & Upper & Wald Chi-Square & df & Sig. \\
\hline (Intercept) & .086 & .0347 & .017 & .154 & 6.060 & 1 & .014 \\
\hline NRS $12 \mathrm{hrs}$ & .709 & .1601 & .395 & 1.023 & 19.612 & 1 & .000 \\
\hline NRS 24hrs & .480 & .1105 & .264 & .697 & 18.901 & 1 & .000 \\
\hline NRS 48hrs & .276 & .1189 & .043 & .509 & 5.404 & 1 & .020 \\
\hline NRS 72hrs & .046 & .0591 & -.070 & .162 & .607 & 1 & .436 \\
\hline NRS $5^{\mathrm{TH}}$ day & $0^{\mathrm{a}}$ & & & & & & \\
\hline
\end{tabular}

\subsubsection{Complication}

There was no apparent complication in either of the intervention or control group during the follow-up period. However, there were mild to moderate nausea and vomiting, photophobia, and neck stiffness in participants with post-dural puncture headaches where nausea and vomiting were the most frequent complication. The overall incidence of this complication was $38.9 \%$ while the incidence of nausea and vomiting was relatively higher in the bupivacaine group as compared to the normal saline group ( $28.9 \%$ vs $38.8 \%)$.

\section{Discussion}

This double Randomized controlled trial was conducted to investigate the effectiveness of prophylaxis intrathecal normal for prevention of post-dural puncture headache in parturient underwent cesarean section under spinal anesthesia.

Post Dural Puncture Headache is the most common complication of neuraxial anesthesia which has been postulated that it is resulting from intracranial hypotension due to continuous loss of CSF through the dural tear that is mainly related to needle size, shape, type, and less likely risk factor including female gender, young age, pregnancy, low BMI, previous history of PDPH, and history of migraine headache $(4,12$, $20,24,38,42,43)$. In turn, these causes prolonged bedridden and thrombosis, decrease early breastfeeding and mother-to-child bonding, length of hospital stay, increased psychological distress, and dissatisfaction to the mother and her family $(13,16,43-45)$.

This study showed that the Sociodemographic characteristics, preoperative baseline variables, sensory onset, peak sensory level, degree of motor block, intraoperative maternal hemodynamics, and Apgar scores were comparable between the groups. However, there were clinical and statistical mean differences between the groups on fifth minutes diastolic and the mean arterial blood pressure which 
may be related to less sympathetic blockage among patients with normal saline due to dilution of bupivacaine.

This study revealed that the overall incidence of PDPH is very high which is comparable with other studies $(16,20,38,45-47)$. Other studies showed that the incidence of PDPH is very high among patients with control compared to an epidural and intrathecal normal saline injection unlike this study where there is no significant difference in the incidence of PDPH despite a clinically significant PDPH was observed among the control group in the first $24 \mathrm{hrs}$ period $(20,46)$.

This study showed that the incidence of PDPH among intervention and control groups didn't show a significant difference which is in line with other randomized control trials and systematic review and meta-analysis $(16,34,45)$. However, other studies did show a significant reduction of PDPH among patients who received prophylactic intrathecal normal saline $(46,47)$. This discrepancy may be related to heterogeneity in the study population, variation in the type, size, and shape of spinal needle, volume of prophylactic normal saline.

In this, study patients experienced mild to moderate pain which was managed with conservative management, acetaminophen, Ibuprofen, and tramadol, and no severe pain was reported. The study revealed that the mean severity score was the highest for the first 48hrs and decreasing thereafter in both intervention and control groups which are comparable with other randomized trials $(43,47,48)$.

In this study, a generalized estimating equation was used after adjusting the NRS pain score to explore the interaction in each repeated measurement. As a result, the GEE model revealed that the NRS pain score was $0.7,0.4$, and 0.2 units higher at 12,24 , and 48 respectively in the Bupivacaine group as compared to the Normal Saline group.

There were mild to moderate nausea and vomiting, photophobia, and neck stiffness in participants with post-dural puncture headache where nausea and vomiting were the most frequent complication which is comparable to other studies conducted globally on this topic.

\subsection{Strength of the study}

This study is a double-blind randomized trial with a relatively large sample size with a long duration of flow up which is about five days postoperatively. The study also tried to investigate the postulated adverse effects of prophylactic intrathecal normal saline including high spinal block, low motor block, and blindness related to rapid injection and obstruction of retinal smaller blood vessels.

\subsection{Limitation of the study}

The study was started before the COVID-19 pandemic which caused many problems where the patient flow was very low, hyperbaric bupivacaine and smaller spinal needle were not available during the lockdown. Besides, we had no PCR machine for diagnosis of COVID-19 and some of the patients might have the disease where the accurate diagnosis of PDPH was a challenge. 


\subsection{Implication for practice}

The independent predictor of PDPH is the spinal needle size, type, and shape but it could happen even with smaller spinal needles and it is inevitable in accidental dural puncture during epidural anesthesia. PDPH is a common complication of neuraxial anesthesia which has a great impact on mother, newborn, family, and health care system particularly in patients with moderate and severe PDPH tolerant to conservative management, because the current treatment and preventive modalities are not conclusive and innovative research on preventive strategy is in demand.

\subsection{Implication for further research}

The study showed a clinical benefit of prophylactic intrathecal normal saline for the prevention of PDPH at least for $48 \mathrm{hrs}$. However, this study used $5 \mathrm{ml}$ normal saline where some of the patients might have COVID-19 and /or more likely vulnerable to different psychological distress which limited our inferences to the general population. The authors recommend further multi-center randomized clinical trials placebo, $5 \mathrm{ml}$, and $10 \mathrm{ml}$ normal saline for surgery under spinal and epidural anesthesia.

\section{Conclusion}

Prophylactic Intrathecal administration of normal saline for prevention of PDPH in patients undergoing cesarean delivery under spinal anesthesia is a safe and simple technique. This study failed to show the effectiveness of prophylactic intrathecal normal saline for the prevention of PDPH for a prolonged period of up to a week and further studies are required to provide a firm conclusion. However, prophylactic intrathecal normal saline could be an option in a resource-limited setup where the appropriate spinal needle is not accessible and management of moderate and severe PDPH is not feasible. We are not recommending the routine use of normal saline for the prevention of PDPH in a setting where small spinal needles are accessible.

\section{Declarations}

\section{Ethics approval and consent to participate}

Ethical clearance and approval were obtained from the ethical review board of the College of Health Science and Medicine.

\section{Consent for publication}

Not applicable

\section{Availability of data and materials}

Data and material can be available where appropriate. 
The authors declare that there are no competing interests

\section{Funding}

This research was funded by Dilla University with a total amount of 65,480 ETB

\section{Acknowledgments}

The authors would like to acknowledge Dilla University for financial support and encouragement to carry out the project.

\section{References}

1. Gibbons L, Belizán JM, Lauer JA, Betrán AP, Merialdi M, Althabe F. The global numbers and costs of additionally needed and unnecessary caesarean sections performed per year: overuse as a barrier to universal coverage. World health report. 2010;30(1):1-31.

2. Robson SJ, De Costa CM. Thirty years of the World Health Organization's target caesarean section rate: time to move on. Medical Journal of Australia. 2017;206(4):181-5.

3. Afolabi BB, Lesi FE. Regional versus general anesthesia for caesarean section. Cochrane database of systematic reviews. 2012(10).

4. Fuzhou W. Gabor Racz, editor. Post dural puncture headache-We can prevent it, pain, and treatment. InTech. 2014;3(2):134-36.

5. Harrison MS, Goldenberg RL. Cesarean section in sub-Saharan Africa. Maternal health, neonatology, and perinatology. 2016;2(1):1-10.

6. Yaya S, Uthman OA, Amouzou A, Bishwajit G. Disparities in caesarean section prevalence and determinants across sub-Saharan Africa countries. Global health research and policy. 2018;3(1):1-9.

7. Akdemir MS, Kaydu A, Yanlı Y, Özdemir M, Gökçek E, Karaman H. The postdural puncture headache and back pain: the comparison of 26-gauge atraucan and 26-gauge quincke spinal needles in obstetric patients. Anesthesia, essays and researches. 2017;11(2):458.

8. Arevalo-Rodriguez I, Ciapponi A, i Figuls MR, Muñoz L, Cosp XB. Posture and fluids for preventing post-dural puncture headache. Cochrane Database of Systematic Reviews. 2016(3).

9. Arevalo-Rodriguez I, Muñoz L, Godoy-Casasbuenas N, Ciapponi A, Arevalo JJ, Boogaard S, et al. Needle gauge and tip designs for preventing post-dural puncture headache (PDPH). Cochrane Database of Systematic Reviews. 2017(4).

10. Babu DD, Chandar DD, Prakash C, Balasubramanian S, Kumar KS. Evaluation of Post Dural Puncture Headache Using Various Sizes of Spinal Needles. Int J Sci Stud. 2015;3(9):9-13.

11. Bezov D, Ashina S, Lipton R. Post-dural puncture headache: Part II-prevention, management, and prognosis. Headache: The Journal of Head and Face Pain. 2010;50(9):1482-98.

12. Bezov D, Lipton RB, Ashina S. Post-dural puncture headache: part I diagnosis, epidemiology, etiology, and pathophysiology. Headache: The Journal of Head and Face Pain. 2010;50(7):1144-52. 
13. Guglielminotti J, Landau R, Li G. Major neurologic complications associated with postdural puncture headache in obstetrics: a retrospective cohort study. Anesthesia \& Analgesia. 2019;129(5):1328-36.

14. Jabbari A, Alijanpour E, Mir M. Post spinal puncture headache, an old problem and new concepts: review of articles about predisposing factors. Caspian journal of internal medicine. 2013;4(1):595.

15. Omole OB, Ogunbanjo GA. Postdural puncture headache: evidence-based review for primary care. South African Family Practice. 2015;57(4):241-6.

16. Bradbury C, Singh S, Badder S, Wakely L, Jones P. Prevention of post-dural puncture headache in parturients: a systematic review and meta-analysis. Acta Anaesthesiologica Scandinavica. 2013;57(4):417-30.

17. Gisore E, Mung'ayi V, Sharif T. Incidence of post dural puncture headache following caesarean section under spinal anaesthesia at the Aga Khan University Hospital, Nairobi. East African medical journal. 2010;87(6):227-30.

18. Kassa AA, Beyen TK, Denu ZA. Post dural puncture headache (PDPH) and associated factors after spinal anesthesia among patients in University of Gondar Referral and Teaching Hospital, Gondar, North West Ethiopia. 2015.

19. Kracoff SL, Kotlovker V. Post dural puncture headache-review and suggested new treatment. Open Journal of Anesthesiology. 2016;6(09):148.

20. Kuczkowski K, Benumof J. Decrease in the incidence of post-dural puncture headache: maintaining CSF volume. Acta anaesthesiologica scandinavica. 2003;47(1):98-100.

21. Russell R, Laxton C, Lucas D, Niewiarowski J, Scrutton M, Stocks G. Treatment of obstetric post-dural puncture headache. Part 1: conservative and pharmacological management. International journal of obstetric anesthesia. 2019;38:93-103.

22. Tafesse D, Melkamayew A. Magnitude of post dural puncture headache and associated factors in obstetric mothers undergone spinal anesthesia for caesarean section. J of Anes \& Cri Open Access. 2019;11(2):46-50.

23. Tarekegn F, Eshetie S, Aregawi A, Moges K. Assessment of the Prevalence and Associated Risk Factors of Post Dural Puncture Headache (PDPH) after Cesarean Section Delivery under Spinal Anesthesia. J Anesth Crit Care Open Access. 2017;8(6):00330.

24. Wu CL, Rowlingson AJ, Cohen SR, Michaels RK, Courpas GE, Joe EM, et al. Gender and post-dural puncture headache. The Journal of the American Society of Anesthesiologists. 2006;105(3):613-8.

25. Peralta F, Higgins N, Lange E, Wong CA, McCarthy RJ. The relationship of body mass index with the incidence of postdural puncture headache in parturients. Anesthesia \& Analgesia. 2015;121(2):451-6.

26. Geracioti Jr TD. Cigarette smokers have reduced risk for post-dural puncture headache. Pain physician. 2013;16:E25-E30.

27. Ansaloni L, Balzani C, Falaschi F, Pazè E. Post-spinal headache after dural puncture with perpendicular or horizontal needle bevel direction: a randomized controlled trial in an African rural hospital. Tropical doctor. 2000;30(3):167-9. 
28. Imarengiaye C, Ekwere I. Postdural puncture headache: a cross-sectional study of incidence and severity in a new obstetric anaesthesia unit. African journal of medicine and medical sciences. 2006;35(1):47-51.

29. Mohammed A, Ayyuba R, Salisu I, Nagoma A, Owolabi L, Ibrahim A. An analysis of postdural puncture headache in obstetric patients: A study from Kano, Nigeria. Tropical Journal of Obstetrics and Gynaecology. 2017;34(1):16-20.

30. Nafiu OO, Salam RA, Elegbe EO. Post dural puncture headache in obstetric patients: experience from a West African teaching hospital. International journal of obstetric anesthesia. 2007;16(1):4-7.

31. Nuhu SI, Embu HY, Shambe I. Prevalence of Postdural Puncture Headache among Caesarean Section Patients in North Central Nigeria. 2017.

32. Abdelgalel EF, Nofal OA, Shahin MA. Postdural puncture headache and maternal hemodynamics after single-shot and continuous spinal anesthesia using epidural kit for cesarean section. Research and Opinion in Anesthesia and Intensive Care. 2018;5(1):35.

33. Krivak TC, Zorn KK. Venous thromboembolism in obstetrics and gynecology. Obstetrics \& Gynecology. 2007;109(3):761-77.

34. Apfel C, Saxena A, Cakmakkaya O, Gaiser R, George E, Radke O. Prevention of postdural puncture headache after accidental dural puncture: a quantitative systematic review. British journal of anaesthesia. 2010;105(3):255-63.

35. Boonmak P, Boonmak S. Epidural blood patching for preventing and treating post-dural puncture headache. Cochrane Database of Systematic Reviews. 2010(1).

36. Esmail S, Salih A, Esmail MM. Epidural Blood Patch as a Treatment Intervention in Post-Dural Puncture Headache: A Systematic Review of Randomised-Controlled Trials.

37. Niraj G, Kelkar A, Girotra V. Greater occipital nerve block for postdural puncture headache (PDPH): a prospective audit of a modified guideline for the management of PDPH and review of the literature. Journal of clinical anesthesia. 2014;26(7):539-44.

38. Patel R, Urits I, Orhurhu V, Orhurhu MS, Peck J, Ohuabunwa E, et al. A comprehensive update on the treatment and management of postdural puncture headache. Current pain and headache reports. 2020;24(6):1-9.

39. Charsley MM, Abram SE. The injection of intrathecal normal saline reduces the severity of postdural puncture headache. Regional Anesthesia and Pain Medicine. 2001;26(4):301-5.

40. Schulz KF, Altman DG, Moher D. CONSORT 2010 statement: updated guidelines for reporting parallel group randomised trials. Trials. 2010;11(1):1-8.

41. Craig D, Carli F. Bromage motor blockade score-a score that has lasted more than a lifetime. Canadian Journal of Anesthesia/Journal canadien d'anesthésie. 2018;65(7):837-8.

42. Ali HM, Mohamed MY, Ahmed YM. Postdural puncture headache after spinal anesthesia in cesarean section: Experience in six months in 2736 patients in Kasr El aini teaching hospital-Cairo University. Egyptian Journal of Anaesthesia. 2014;30(4):383-6. 
43. Turnbull D, Shepherd D. Post-dural puncture headache: pathogenesis, prevention and treatment. British journal of anaesthesia. 2003;91(5):718-29.

44. Orbach-Zinger S, Eidelman LA, Livne MY, Matkovski O, Mangoubi E, Borovich A, et al. Long-term psychological and physical outcomes of women after postdural puncture headache: A retrospective, cohort study. European Journal of Anaesthesiology| EJA. 2021;38(2):130-7.

45. Suescun H, Austin P, Gabaldon D. Nonpharmacologic Neuraxial Interventions for Prophylaxis of Postdural Puncture Headache in the Obstetric Patient. AANA journal. 2016;84(1).

46. Tazeh-Kand NF, Eslami B, Marzony SG, Abolhassani R, Mohammadian K. Injection of intrathecal normal saline in decreasing postdural puncture headache. Journal of anesthesia. 2014;28(2):206-9.

47. Uludağ Ö, Tutak A. Efficacy of prophylactic epidural saline for reducing postdural puncture headache in patients undergoing caesarean section. Journal of Surgery and Medicine. 2019;3(8):593-7.

48. Kiki I, Gundogdu M, Alici HA, Yildirim R, Bilici M. A simple, safe and effective approach to prevent postdural puncture headache: epidural saline injection. The Eurasian journal of medicine. 2009;41(3):175.

\section{Figures}




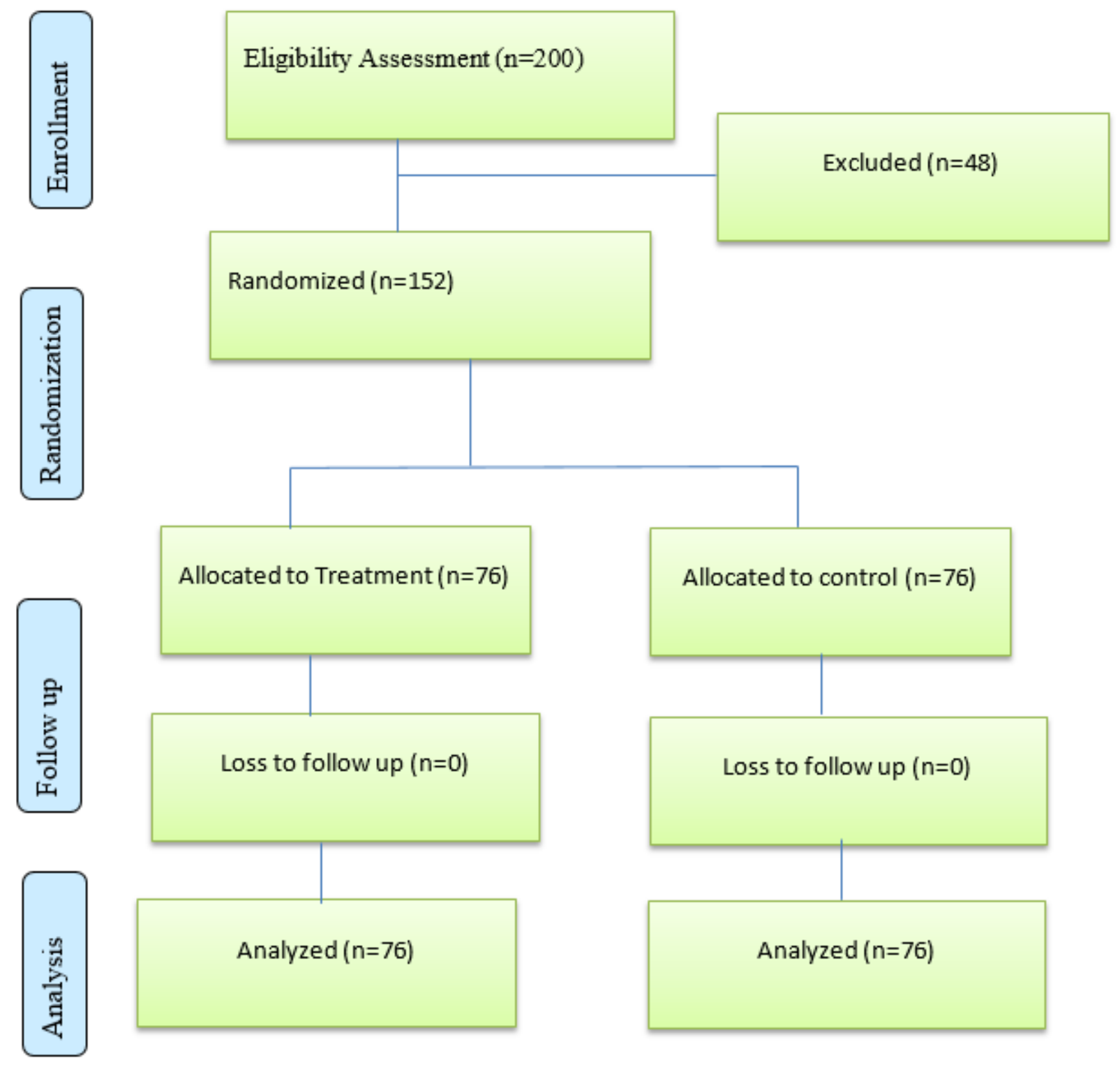

Figure 1

consort flow diagram 


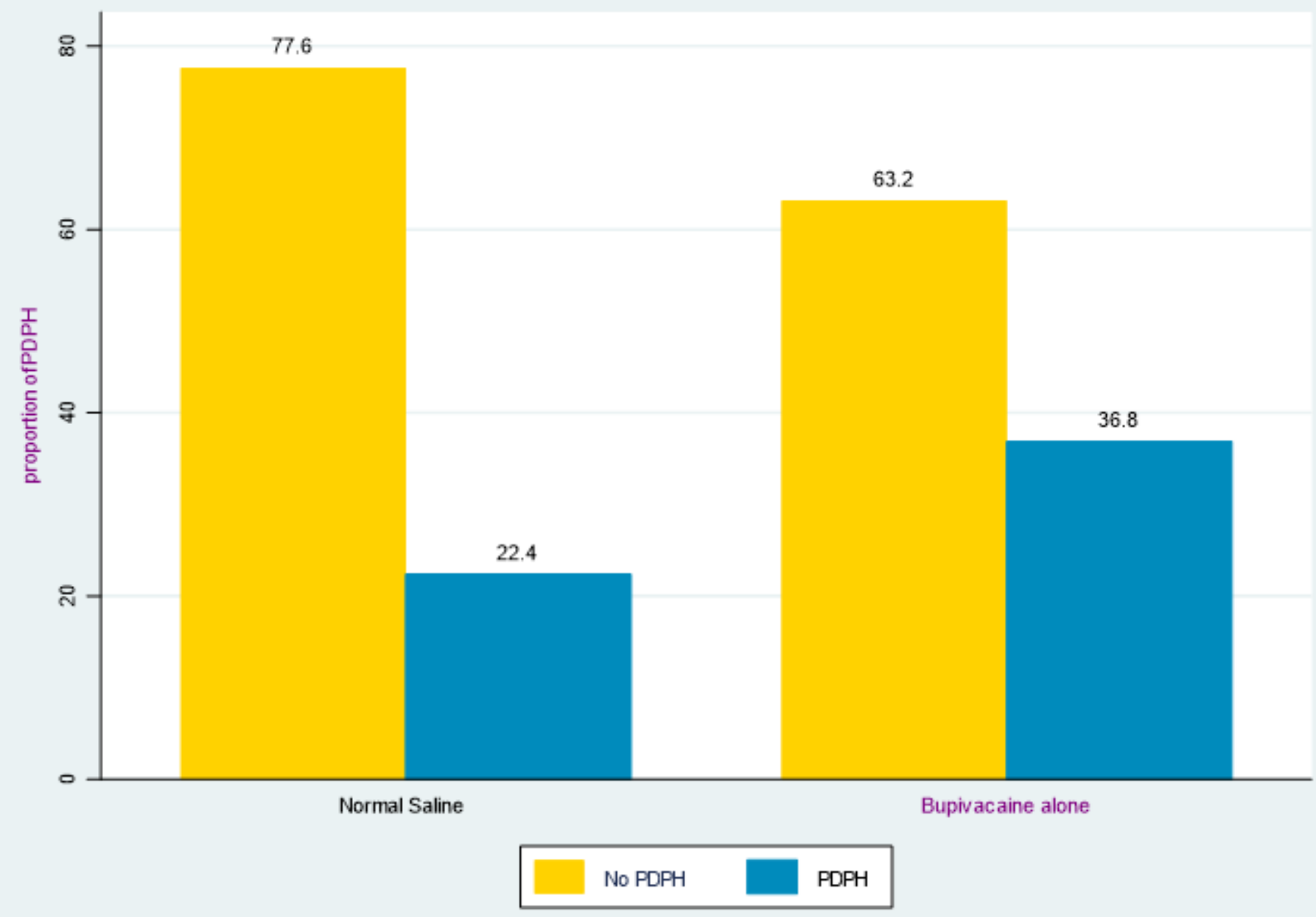

Figure 2

Incidence of PDPH among patients who underwent cesarean section under spinal anesthesia with bupivacaine alone and bupivacaine with 5ml Normal Saline in Dilla University Referral Hospital, 2021. 


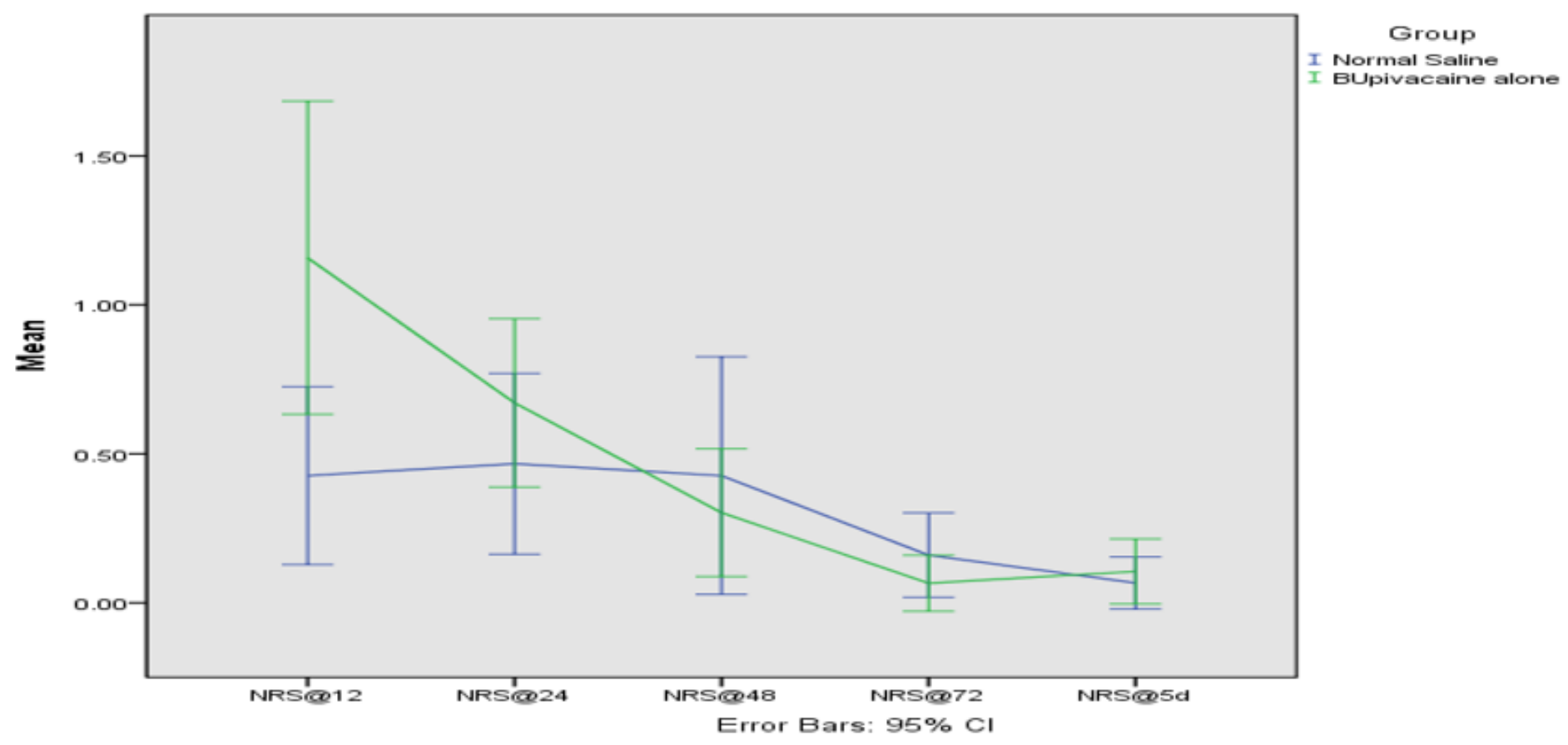

Figure 3

Mean severity of PDPH at each follow-up period among participants who underwent cesarean section under spinal anesthesia in Dilla University referral hospital, Ethiopia, 2021.

\section{Supplementary Files}

This is a list of supplementary files associated with this preprint. Click to download.

- CONSORT2010Checklist.doc

- suplemetntaltable.docx 